\title{
Confinement Improvement with Magnetic Levitation of Superconducting Dipole
}

\author{
D.T. Garnier 1), A.C. Boxer 2), J.L. Ellsworth 2), J. Kesner 2), M.E. Mauel 1)
}

1) Department of Applied Physics, Columbia University, New York, NY 10027, USA

2) PSFC, Massachusetts Institute of Technology, Cambridge, MA 02139, USA

e-mail contact of main author: dg276@columbia.edu

\begin{abstract}
We report the first production of high beta plasma confined in a fully levitated laboratory dipole using neutral gas fueling and electron cyclotron resonance heating. The pressure results primarily from a population of energetic trapped electrons that is sustained for many seconds of microwave heating provided sufficient neutral gas is supplied to the plasma. As compared to previous studies in which the internal coil was supported, levitation results in improved particle confinement that allows higher-density, high-beta discharges to be maintained at significantly reduced gas fueling. Elimination of parallel losses coupled with reduced gas leads to improved energy confinement and a dramatic change in the density profile. Improved particle confinement assures stability of the hot electron component at reduced pressure. By eliminating supports used in previous studies, cross-field transport becomes the main loss channel for both the hot and the background species. Interchange stationary density profiles, corresponding to an equal number of particles per flux tube, are commonly observed in levitated plasmas.
\end{abstract}

\section{Introduction}

The dipole confinement concept $[1,2]$ was motivated by spacecraft observations of planetary magnetospheres that show centrally-peaked plasma pressure profiles forming naturally when the solar wind drives plasma circulation and heating. Unlike most other approaches to magnetic confinement in which stability requires average good curvature and magnetic shear, MHD stability in a dipole derives from plasma compressibility [3-5]. At marginal stability $\delta\left(p V^{\gamma}\right)=0$ (with $p$ the plasma pressure, $V=\oint d l / B$ is the differential flux tube volume, and $\gamma=5 / 3$ ), and an adiabatic exchange of flux tubes does not modify the pressure profile nor degrade energy confinement. Non-linear studies indicate that large-scale convective cells will form when the MHD stability limit is weakly violated, which results in the circulation of plasma between the hot core and the cooler edge region [6]. Studies have also predicted that the confined plasma can be stable to low frequency (drift wave) modes when $\eta=d \ln$ $\mathrm{T}_{\mathrm{e}} / \mathrm{d} \ln \mathrm{n}_{\mathrm{e}}>2 / 3$ [7]. The marginally stable case to both drift waves and MHD modes, is thus where:

$$
\begin{aligned}
& p \propto V^{\gamma} \text { and } \\
& n \propto V^{-1} .
\end{aligned}
$$

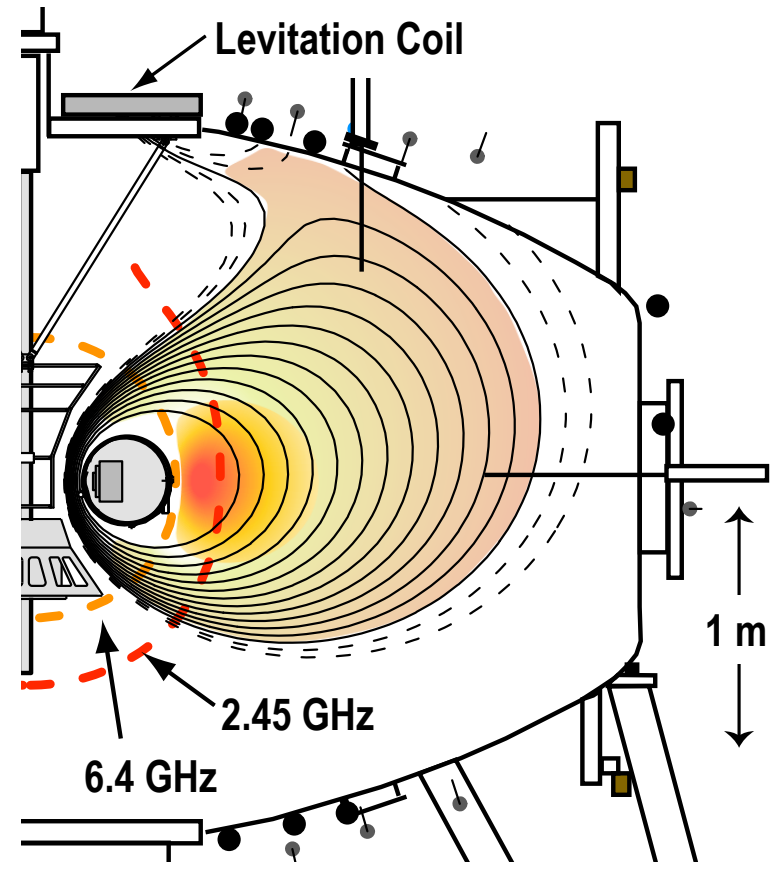

FIG. 1. Schematic of LDX device showing electron cyclotron resonance zones configuration. 
This case corresponds to an equal number of particles and entropy per flux tube. These profiles are the expected result of sufficient interchange mixing [8], and they form the design basis for attractive dipole fusion reactor concepts [9].

The levitated dipole experiment (LDX), shown in Fig. 1, is investigating the confinement and stability of plasma in a dipole magnetic field configuration [10]. In the experiments reported here, high-beta plasma discharges were studied when the LDX high-field superconducting dipole magnet was levitated by attraction to a coil located above the vacuum chamber. The resulting configuration utilizes a digital feedback system to obtain stable levitation of the floating coil. Experiments with the floating coil fully levitated began in 2007. Comparison "supported mode" shots were taken with the cone shaped launching fixture raised to contact the inner section of the dipole and providing a loss mechanism for particles along all field lines.

In many experiments, the plasma was heated by $5 \mathrm{~kW}$ of electron cyclotron resonance heating (ECRH) evenly divided between 2.45 and $6.4 \mathrm{GHz}$. In other experiments, a third microwave source provided an additional $10 \mathrm{~kW}$ of heating at $10.5 \mathrm{GHz}$. This multi-frequency ECRH creates a two component plasma containing a hot electron species. The plasma stored energy is dominated by a population of energetic electrons with $E_{e h}>50 \mathrm{keV}$ and $n_{e h} \sim 10^{16} \mathrm{~m}^{-3}[11]$.

\section{Improved confinement with levitation}

Figure 2 compares typical discharges in the supported and levitated modes of operation. A multi-chord interferometer is utilized to observe the background density profile [12]. For a similar level of fueling resulting in similar vacuum pressure (Fig 2b), we observe in the levitated mode a broader density profile and a higher overall density (Fig. 2c), indicative of improved confinement. In the afterglow, when the ECRH power is removed, a rapid plasma loss is seen in the supported mode as the plasma is lost to the supports. In the levitated operating mode a slow density decay is sometimes seen, matching the slow decay in the energetic particles.

The plasma diamagnetic flux (Fig. 2d), indicative of plasma beta, is doubled in the levitated mode of operation. This plasma stored energy, in supported operation contained in the hot electron population gives an idea of the improvement in confinement of the

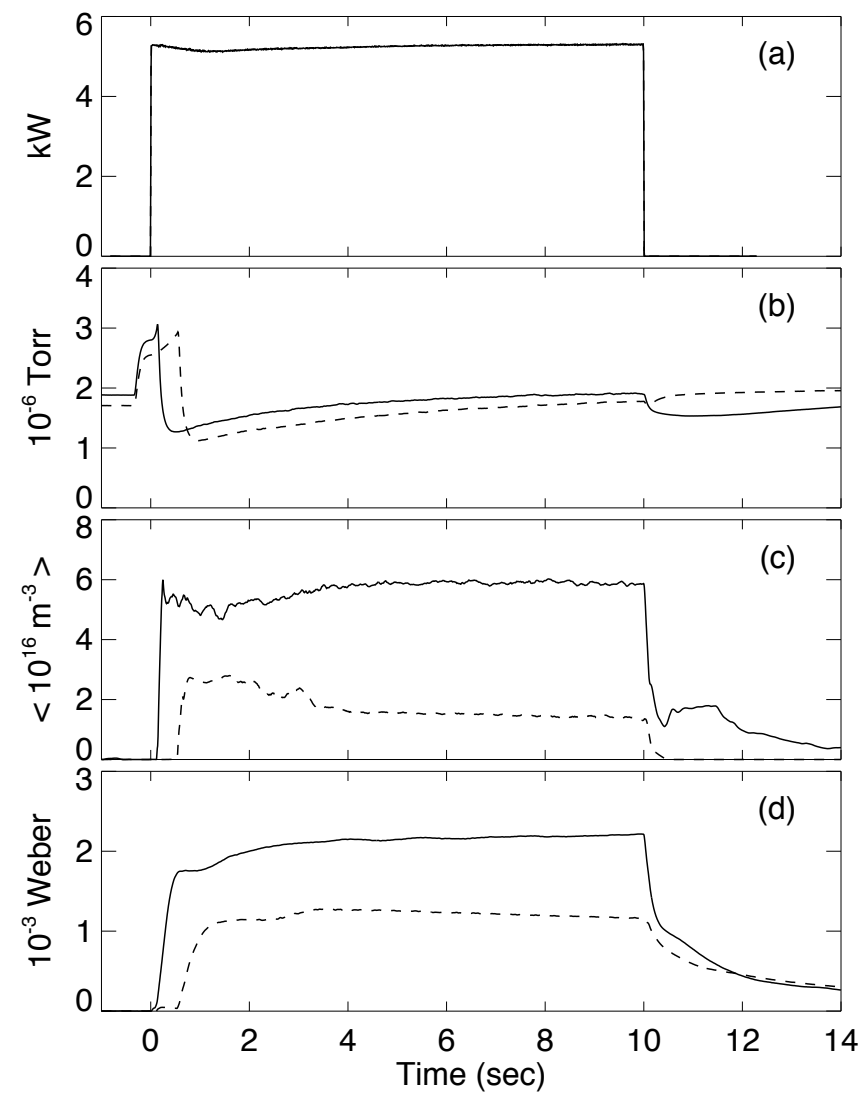

FIG. 2. Wave forms for discharge in supported (dashed) and levitated (solid) mode: a) RF power, b) vacuum, $c$ ) $R=77 \mathrm{~cm}$ density chord and d) midplane diamagnetic signal. 
fast electrons which now must also be lost radially. Another indication of this cross field hot electron transport was an observed heating of probes (located close to the plasma separatrix) up to temperatures at which they glow brightly ( $\sim 2000 \mathrm{deg} C)$. This probe heating is not observed in supported experiments.

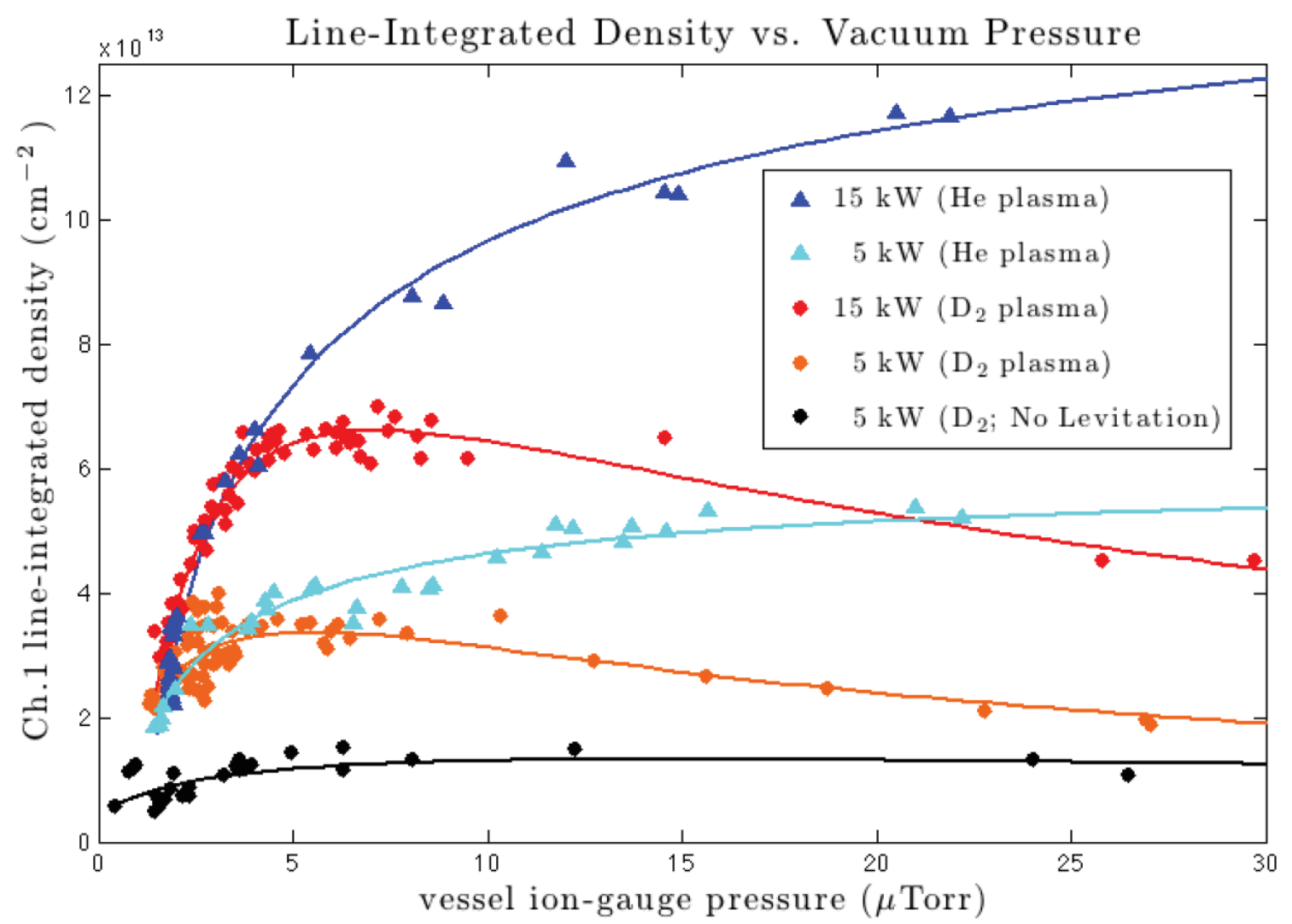

FIG. 3: Steady state central chordal density measurment versus neutral pressure for different conditions. The effect of levitaiton for deuterium plasmas with $5 \mathrm{~kW}$ of input ECRH is shown as increased density by a factor of 2-4. (Also depicted are significant power and species dependencies at high neutral pressure).

Data from a collection of shots with different conditions give a statistical picture of the same improvement in confinement. Shown in Figure 3 is the central chord line density as a function of neutral pressure. While the line average density increases with neutral gas fueling and with increasing microwave power, the data show a 2-3 fold increase as the mechanical supports are withdrawn from the plasma. Fig. 3 also shows a positive mass scaling to confinement at higher fueling rates, which we believe indicates the ratelimiting role of edge plasma loss on open field lines beyond the magnetic separatrix.

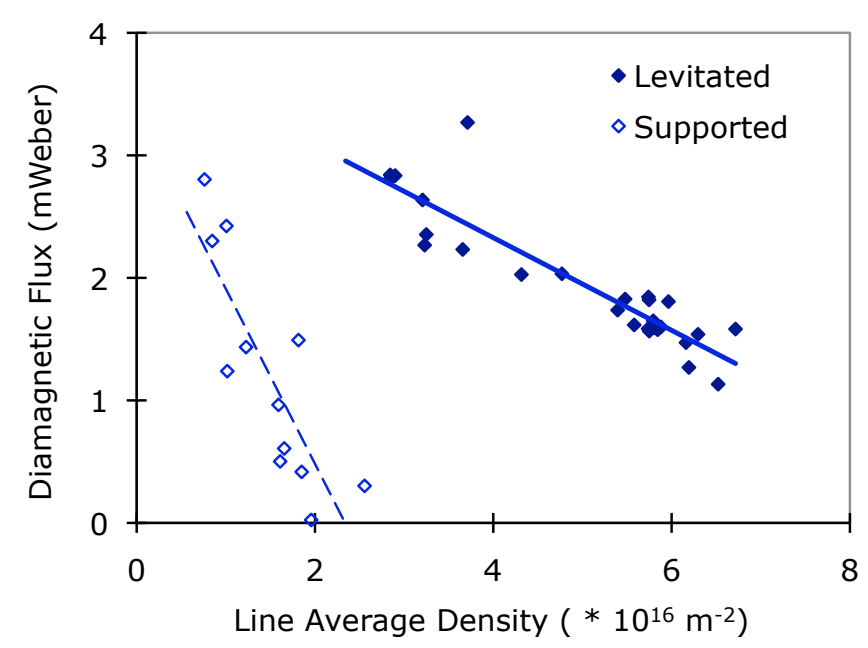

FIG. 4. Diamagnetic flux for supported and levitated operation during density scan of run 80321. Both operations show an inverse scaling between fast particle confinement and bulk density. 
Figure 4 shows the relationship between chord averaged density and diamagnetic flux for levitated and supported plasmas. The inverse relationship previously seen between stored energy and density for supported plasmas is altered for levitation allowing 3 times higher density for the similar diamagnetic flux.

\section{Observation of interchange stationary profiles}

A four channel interferometer [12] was used to monitor the time evolution of the density profile and the change in density profile resulting from magnetic levitation of the superconducting dipole. One approximate and appropriate method to reconstruct the density profile from the line density measured by the interferometer array is to compare the measured line densities to that which would be obtained if $n \propto 1 / V$. When $n \propto$ $1 / V$, then the ratio of line density measurements take specific values, $P_{i}$, determined by known geometry. A deviation from $P_{i}$ occurs when the profile is more or less steep from the the marginal profile. We define the global deviation from the marginal density profile as $\Delta \propto \sum\left(R_{i}-P_{i}\right)^{2}$, an RMS average of the individual line-density ratios, or simply a density profile deviation parameter. When $\Delta$ is small, then the density profile is well described as $n \propto 1 / V$. When $\Delta>0$, then the profile is approximately described as $n \propto 1 / V^{ \pm \Delta}$. Figure 5 shows measurements of density profile deviation parameter for highpower discharges as a function of neutral gas pressure. Except for the lowest pressures, we observe the density profile is always well described by the marginal profile when the superconducting dipole is levitated.

When the mechanical supports are reinserted into the plasma, the central density profile collapses signifying significant particle loss along open field lines. Figure 6 compares the density profile from two similar discharges at

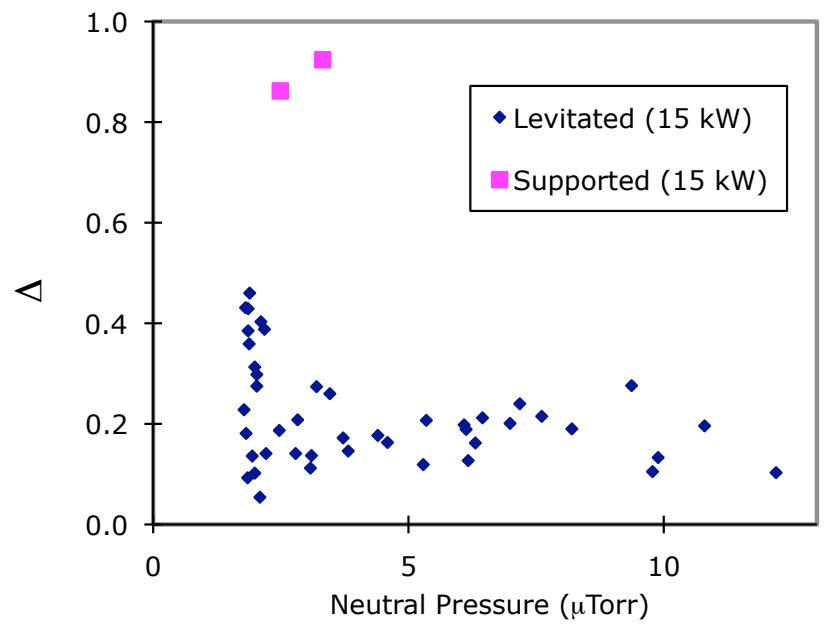

FIG. 5: Deviation parameter (4) from constant number of particles per flux tube profile. Levitated plasmas with multi-source ECRH and sufficient neutral fueling exhibit close to interchange stationary profiles.

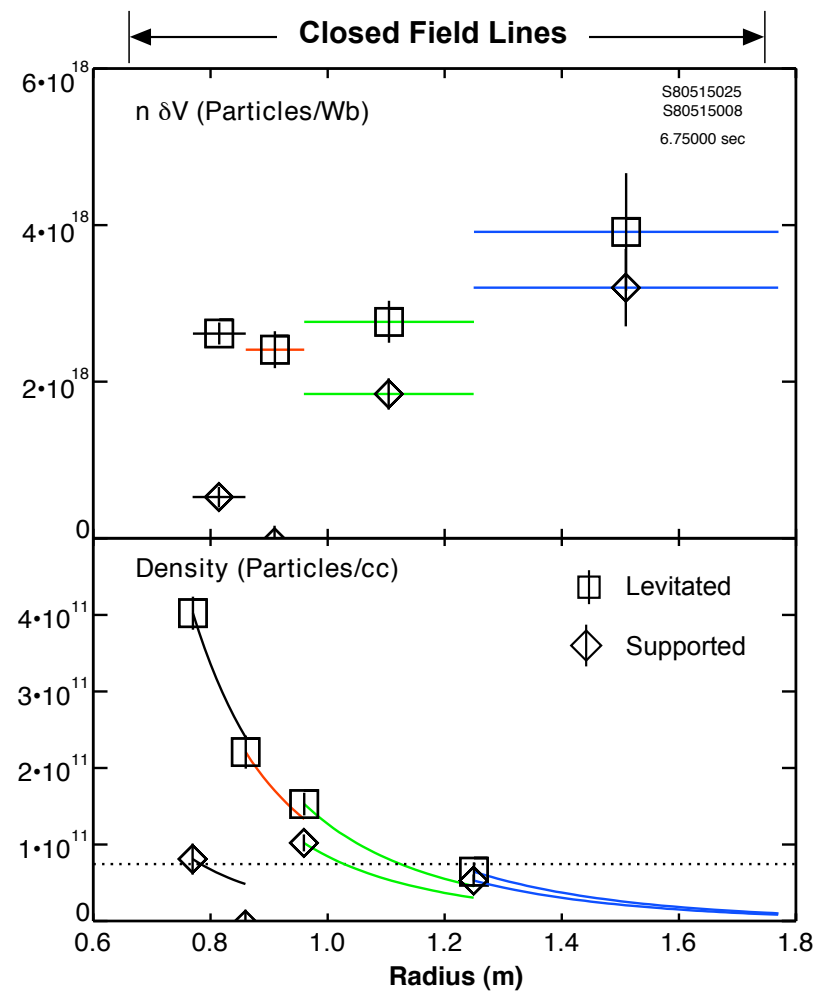

FIG. 6: Computation of the density profile for supported (diamond) and levitated (square) discharges under similiar conditions with $15 \mathrm{~kW}$ of ECRH. 
$15 \mathrm{~kW}$ ECRH power. When the dipole coil is levitated, the central density is highly peaked and at least four-times larger than the central density obtained with a supported dipole. With supports, the density profile is hollow, indicative of the parallel loss of plasma between radially localized source regions.

\section{Low frequency fluctuations in levitated plasmas}

Plasma confinement with a levitated dipole is dominated by radial transport, and levitated experiments have permitted the exploration of the dynamics and transport of plasma confined in a magnetic configuration relevant to a potential dipole fusion reactor. The edge background plasma in LDX has $n_{e} \sim 0.2-5 \times 10^{16} \mathrm{~m}^{-3}$ and $T_{e} \sim 10-20 \mathrm{eV}$, and we observe fluctuations in the diamagnetic drift frequency range $(0.2-10 \mathrm{kHz})$ that are effected by the rate of neutral fueling. Similarly to previous observations [13], large scale and low toroidal wave number are observed. In levitated plasmas, the modes exhibit a radial structure with different frequencies seen across the discharge. These low-frequency fluctuations co-exist with the high-beta fast electrons but are most probably associated with convective and/or drift-wave-like modes of the cooler background plasma. For typical discharges, like those shown in Figs. 2-6, lowfrequency fluctuations are always observed and may indicate the nature of the plasma electrostatic interchange-like convection that is responsible for peaked profiles with $n \propto 1 / V$.

\section{Improved stability to HEI}

The hot electron species can be unstable to the hot electron interchange (HEI) [14-16] mode when the hot electron density gradient is sufficiently high and the background density is sufficiently low [10]. Similarly to supported mode operation, when the dipole is levitated the plasma entered a high-density regime in which the HEI was stabilized for sufficient gas fueling. However, the plasma was maintained in the high-density regime when the gas fueling was substantially reduced as compared to the supported mode. In fact, we have not yet observed a case in levitated operation when high-beta discharges are subject to fast, largescale instability. Additionally, limiting HEI activity is not observed during dipole levitation, and the HEI remained stable at all operating pressures. We believe the enhanced stability of the HEI during levitation results from both a broadened hot electron profile and a higher plasma density resulting from improved particle confinement.

\section{Summary}

We report the first production of high beta plasma confined by a levitated superconducting dipole magnet. As compared to previous studies in which the internal coil was supported [11], levitation results in improved particle confinement that allows high-density, high-beta discharges to be maintained at significantly reduced gas fueling. Elimination of parallel losses coupled with reduced gas leads to improved energy confinement and a dramatic change in the density profile. Improved particle confinement assures stability of the hot electron component at reduced pressure. By eliminating supports used in previous studies, cross-field transport becomes the main loss channel for both the hot and the background species. Four-channel microwave interferometry show the density profile during magnetic levitation to be characterized by nearly an equal number of particles per flux tube, $n \propto 1 / V$. 


\section{Acknowledgments}

This work was supported by US DOE grants DE-FG02-98ER54458 and DEFG02-98ER54459.

\section{References}

[1] HASEGAWA, A., "A Dipole Field Fusion Reactor". Comments Plasma Phys., 11, (1987), 147-51.

[2] HASEGAWA, A, CHEN, L., MAUEL, M.E., "A D- ${ }^{3} H e$ fusion-reactor based on a dipole magnetic-field", Nucl. Fusion 30, (1990) 2405.

[3] ROSENBLUTH, M.N., and LONGMIRE, C.L. "Stability of Plasmas Confined by Magnetic Fields". Ann. Phys., 1 (1957), 120-40.

[4] GOLD, T. "Motions in the Magnetosphere of the Earth". J. Geophys. Res., 14, (1959), 1219-24.

[5] GARNIER, D.T., KESNER, J., and MAUEL, M.E., "Magnetohydrodynamic stability in a levitated dipole", Phys. Plasmas 6, 3431 (1999).

[6] PASTUKHOV, V.P. and CHUDIN, N.V., "Plasma Convection near the Threshold for MHD Instability in Nonparaxial Magnetic Confinement Systems", Plasma Phys. Reports, 27, (2001), 963-77.

[7] KESNER, J.. HASTIE, R.J., "Electrostatic drift modes in a closed field line configuration" Phys. Plasmas 9, (2002) 4414.

[8] KOUZNETSOV, A., FREIDBERG, J.P., KESNER, J., "Quasilinear theory of interchange modes in a closed field line configuration". Phys. Plasmas, 14, (2007), 102501.

[9] KESNER, J, et al., "Helium catalysed D-D fusion in a levitated dipole," Nucl. Fusion, 44, (2004), 193-203.

[10] GARNIER, D.T., et al., "Design and initial operation of the LDX facility", Fusion Eng. Des., 81, (2006) 2371-80.

[11] GARNIER, D.T., et al., "Production and study of high-beta plasma confined by a superconducting dipole magnet", Phys. Plasmas 13, (2006) 056111.

[12] BOXER, A., Density Profiles of Plasmas Confined by the Field of a Levitating Dipole Magnet, PhD Thesis, Massachussetts Institute of Technology (2008).

[13] GARNIER, D.T., et al., "Stabilization of a low-frequency instability in a dipole plasma", J. Plasma Phys, 74, (2008) doi:10.1017/S0022377808007071.

[14] KRALL, N.A., "Stabilization of Hot Electron Plasma by a Cold Background", Phys. Fluids, 9, (1966) 820.

[15] LEVITT, B., MASLOVSKY, D., MAUEL, M., "Measurement of the global structure of interchange modes driven by energetic electrons trapped in a magnetic dipole", Phys. Plasmas, 9, (2002), 2507.

[16] KRASHENINNIKOVA, N., CATTO, P., "Effects of hot electrons on the stability of a closed field line plasma", Phys. Plasmas, 12, (2005) 32101. 